\title{
Correction to: detection of Cryptosporidium and Giardia in the slaughterhouse, sewage and river waters of the Qinghai Tibetan plateau area (QTPA), China
}

\author{
Liqing $\mathrm{Ma}^{1} \cdot$ Xueyong Zhang ${ }^{1} \cdot$ Yingna Jian $^{1} \cdot$ Xiuping $\mathrm{Li}^{1} \cdot$ Geping Wang ${ }^{1} \cdot$ Yong Hu$^{1} \cdot$ Panagiotis Karanis ${ }^{1,2}$
}

Published online: 14November 2019

(C) Springer-Verlag GmbH Germany, part of Springer Nature 2019

\section{Correction to: Parasitology Research 118(7): 2041-2051}

https://doi.org/10.1007/s00436-019-06330-w

The authors of this article would like to state that $C$. environmental is not a species, but rather a group of un-identified Cryptosporidium isolates from the environment. It is referred to in the literature as Cryptosporidium environmental sequence and not as a species.

The online version of the original article can be found at https://doi.org/ 10.1007/s00436-019-06330-w

\section{Panagiotis Karanis}

panagiotis.karanis@uk-koeln.de

1 Qinghai Academy of Animal Sciences and Veterinary Medicine,

Centre for Biomedicine and Infectious Diseases, Qinghai University,

State Key Laboratory of Plateau Ecology and Agriculture,

Xining 810016, People's Republic of China

2 Medical Faculty and University Hospital Cologne, University of

Cologne, Cologne, Germany 